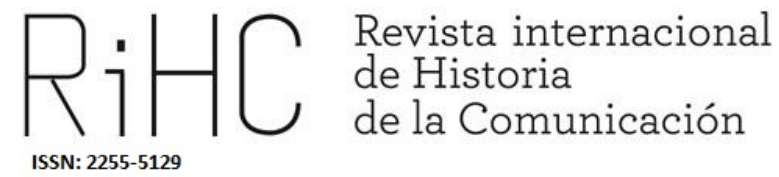

\title{
ANÁLISIS DE LAS RELACIONES ENTRE MEDIOS DE COMUNICACIÓN DIGITALES: EJEMPLOS DEL PANORAMA ESPAÑOL
}

Analysis of the relations among digital media: examples of the Spanish panorama

DOI: http://dx.doi.org/10.12795/RiHC.2019.i13.04

Recibido: $14 / 10 / 2019$

Aceptado: 28/11/2019

Publicado: $15 / 12 / 2019$

Alicia De Lara González

ORCID (D) 0000-0001-5556-6317

Universidad Miguel Hernández de Elche, España

a.lara@umh.es

Lorena Santos Maestre

ORCID (iD) 0000-0002-5349-6914

Universidad Miguel Hernández de Elche, España

Isantos@umh.com

Como citar este artículo: DE LARA GONZÁLEZ, Alicia y SANTOS MAESTRE, Lorena (2019): "Análisis de las relaciones entre medios de comunicación digitales: ejemplos del panorama español”, en Revista Internacional de Historia de la Comunicación (13) , 53. 70. 
Resumen: El panorama nacional de medios digitales cuenta con multitud de ejemplos en los que las iniciativas interactúan unas con otras e incluso crean proyectos de forma conjunta. Ante esta tendencia a la colaboración, propia de los proyectos que nacen en la red, la presente investigación analiza las colaboraciones y asociaciones que se han producido entre las iniciativas periodísticas españolas consideradas como más innovadoras, de acuerdo a dos proyectos de referencia: el Ranking de Innovación Periodística, elaborado por la Universidad Miguel Hernández de Elche y a la web del Observatorio de Nuevos Medios, coordinada por un equipo de especialistas en diversas áreas. A través de una selección de entrevistas a los responsables de algunos de los medios, del estudio y análisis de contenido de sus plataformas y de documentos y páginas web relacionadas con el panorama mediático nacional, se estudia la simbiosis entre diferentes medios para la creación de proyectos con más o menos continuidad; la absorción de unos por otros o las colaboraciones puntuales para la puesta en marcha de nuevos formatos.

Palabras clave: innovación, periodismo, colaboración, medios de comunicación, competencia

\begin{abstract}
The national digital media landscape has many examples in which initiatives interact with each other and even create projects together. Given this trend of collaboration, typical of the projects that are born in the network, this research analyzes the collaborations and associations that have taken place between the Spanish journalistic initiatives considered as more innovative, according to two reference projects: the Ranking of Innovation Journalism, prepared by the Miguel Hernández University of Elche and the New Media Observatory website, coordinated by a team of specialists in various areas. Through a selection of interviews with those responsible for some of the media, the study and analysis of the content of their platforms and of documents and web pages related to the national media landscape, the symbiosis between different means for the creation of projects is studied. with more or less continuity; the absorption of some by others or the punctual collaborations for the start-up of new formats.
\end{abstract}

Keywords: innovation, journalism, collaboration, media, competition

\title{
1 Marco teórico
}

El informe elaborado por la Universidad de Oxford y el Instituto Reuters (Borchardt et al., 2019) sobre las necesidades en las redacciones de los medios en tres países europeos revela que existe una importante preocupación entre los líderes de los medios por encontrar y atraer la combinación correcta de talento para acceder a diferentes audiencias e inspirar su confianza. La investigación descubrió asimismo que existe una notable falta de diversidad en las redacciones de los medios, lo que indica que es necesario mejorar los procesos de reclutamiento y la definición de los equipos de trabajo. 
Ante un panorama mediático en continua transformación, los trabajos sobre cómo el desarrollo de internet ha revolucionado la industria periodística se publican desde hace ya más de una década, son prolíficos y adoptan enfoques y perspectivas diferentes. Vemos cómo la red ha transformado la producción de los contenidos (Canavilhas, 2009; Cebrián Herreros, 2010; García-Avilés, 2016); los cambios que conciernen a las audiencias (Flores Vivar, 2009; Túñez, 2009; Meso Ayerdi: 2013) o la transformación de la organización de las redacciones (Domingo, 2005; Puerta, 2006; Meier, 2010; García-Santamaría, Fernández y López-Aboal, 2013 ). ${ }^{1}$

Todas estas investigaciones han demostrado cómo las tecnologías digitales han traído consigo un enriquecimiento de la actividad periodística. Como explicaba Salaverría (2009: 3) hace ahora una década:

Con la entrada del nuevo siglo, las organizaciones periodísticas se enfrentan a un contexto en el que las reglas de juego han cambiado sustancialmente. Desde los primeros pasos del proceso de producción hasta las etapas finales de la difusión y comercialización de los contenidos periodísticos, todos los eslabones de la cadena productiva han experimentado cambios radicales.

De nuevo Salaverría señalaba en uno de sus primeros trabajos sobre Prensa Digital (2006) cuatro "retos pendientes" que consideraba "de especial relevancia para el desarrollo futuro del ciberperiodismo" y que, una década después, continúan siendo un desafío: 1) la exploración de nuevos lenguajes periodísticos para aprovechar las posibilidades comunicativas de la Red; 2) la consolidación de nuevos estándares profesionales para el ejercicio del ciberperiodismo; 3) la instauración de modelos de negocio sostenibles en internet y 4) la formación de los periodistas en el uso de las nuevas tecnologías.

Especialmente, el tercer punto sigue preocupando tanto a los experimentados empresarios como a los nuevos: la búsqueda de un modelo informativo. Pero esta cuestión no se restringe a la producción ni a las audiencias ni a los contenidos. La sostenibilidad del negocio periodístico tiene que ver con todo el proceso y también con el entorno. Hasta el punto de que el propio concepto de innovación se entiende como "una creación de valor añadido para que el producto periodístico aparezca atractivo a los ojos del consumidor, pero también como la búsqueda de un modelo que permita la rentabilidad económica y el ahorro de costes de producción y distribución" (Valero Pastor, 2015).

En la línea de Salaverría y en lo que se refiere a los cambios en el sector empresarial, Becerra ya apuntaba en el año 2000 que se empezaba a apreciar no solo la progresiva diversificación de canales de distribución por parte de los grandes grupos de

\footnotetext{
${ }^{1}$ Una versión previa de este texto fue presentada al XVI Congreso de AsHisCom de Santiago de Compostela.
} 
comunicación, sino también la cooperación entre empresas de medios más modestas y la cooperación de iniciativas periodísticas con otras organizaciones de sectores distintos (Becerra, 2000). La utilización de recursos ajenos, como los de los propios usuarios, implica una nueva disminución de los costes, que para las organizaciones supone la distribución de contenidos en Internet basados en el uso de recursos que no son propios (García-Avilés, Salaverría y Masip, 2008). En un principio, los cambios en cuanto a relaciones empresariales en el panorama mediático se caracterizaron por la concentración empresarial, pero no todos son fruto de la reducción de personal y del descenso del presupuesto y de los recursos. Otros factores, principalmente el papel de la tecnología, influyen en la organización de las nuevas redacciones y en la transformación de las ya consolidadas.

De esta manera, las relaciones que se están generando en la actualidad entre las iniciativas periodísticas digitales no se caracterizan tanto, o no solo, por la concentración o la absorción de unas por otras, sino más bien por la colaboración y la creación de proyectos conjuntos. En este sentido conviene tener en cuenta que analizar la innovación conlleva centrarse no solo en los productos, sino en los procesos que crean los productos y que pueden incorporar innovaciones de modo tangible o intangible (Dogruel, 2013: 57-8). Pero también en las estructuras de las propias empresas informativas quienes, como señala Briggs (2012), están poniendo cada vez más énfasis en los entornos de trabajo flexibles, la investigación y uso innovador de la tecnología.

En el presente artículo nos centramos en describir las relaciones entre los medios, las relaciones con la competencia, y cómo los medios más innovadores establecen alianzas para alcanzar audiencias diversificadas, pero con intereses comunes ante ciertos contenidos. Estudiar las nuevas rutinas de trabajo y la forma de relacionarse entre los profesionales y los medios nos dará pistas sobre un panorama en el que el concepto de "inteligencia colectiva" que apuntaba Jenkins cobra todo su sentido. Una especie de red no solo digital en la que, según el experto, los límites geográficos se diseminan, surgen nuevas formas de comunidad y de afiliaciones, que comparten intereses y necesitan cambiar y evolucionar y pertenecer a otras comunidades. Comunidades que, según el experto y como veremos que sucede entre algunas iniciativas periodísticas, funcionan como lugares para la discusión colectiva, la negociación y el desarrollo.

\subsection{La colaboración como forma de convergencia periodística}

Tal y como se desprende de las conversaciones con los profesionales, "la forma de organizar los equipos se rige tanto por la reducción de costes, como por la necesidad 
de flexibilizar las rutinas de trabajo y la toma de decisiones" (De Lara et al. 2014: 32). Sin embargo, aunque se palpa la transformación, lo cierto es que el peso de la herencia de lo tradicional todavía es considerable. Un aspecto que dejó patente Boczkowski (2006) en su estudio llevado a cabo sobre diferentes salas de redacción online. Según esta investigación, los proyectos web más innovadores implementaron una función editorial estructurada en torno a un enfoque menos tradicional, entendiendo al público como un conjunto de avezados productores de información, mientras que las redacciones online dependientes de sus homólogas impresas se limitaron a trasponer a la red "los modos del periódico impreso".

En su libro, Boczkowski (2006) insiste en que se desdibujan tanto las fronteras entre las organizaciones, como las que separan a las organizaciones del público al cual se dirigen. $Y$ de esta transformación se desprende el término Conexión Comunitaria, que alude a un nuevo sistema de producción de contenidos informativos en los medios online que permite la existencia de múltiples flujos de información, tanto con la audiencia, como con otros medios.

El Informe Anual de la Profesión Periodística contabilizaba en 201512.200 puestos de trabajo perdidos en todos los medios de España durante la crisis. Como se percibe en el número de medios que han cerrado en los últimos años, 375 medios, según los datos recogidos en el Informe Anual de la Profesión Periodística elaborado por la Asociación de la Prensa de Madrid (APM), los cambios más significativos en las redacciones tradicionales, en cuanto a organización, han tenido que ver principalmente con la reducción de personal y el descenso del presupuesto y de los recursos. Asimismo, la situación de los contratados en periodismo y comunicación es notablemente peor que la del conjunto nacional, según el Informe Anual de la Profesión Periodística 2018. Por lo tanto, resulta lógico pensar que las innovaciones que se adopten en este sentido tendrán como principal objetivo la reducción de costes y la flexibilidad de las rutinas de trabajo.

También conviene poner el foco en los cambios que se han producido en cuanto a los lugares y entornos, los posibles avances en relación a la proximidad con las audiencias y las nuevas rutinas de cooperación, ya se trate entre departamentos del propio medio o incluso entre personal de iniciativas periodísticas distintas que trabajen juntas en determinados proyectos. Incluso, como señala Steen Steensen (2009: 826), conviene tener en cuenta factores como la capacidad de liderazgo de los periodistas, algo que a juicio del autor resulta decisivo en el éxito de los procesos de innovación.

Como explica Cabrera González (2010: 51), la colaboración entre iniciativas se concibe como una forma de convergencia y de integración de redacciones. El autor señala: "Se inicia la convergencia empresarial y la integración de redacciones cuando dos o más medios deciden compartir la producción y la explotación de sus contenidos". El autor también alude a la convergencia profesional, que, según detalla, se produce cuando 
"las tareas -antes repartidas entre varios profesionales- comienzan a recaer sobre uno solo y polivalente, es decir, capaz de trabajar para distintos medios".

Parece evidente que la convergencia está reconfigurando las relaciones fundamentales entre periodistas, sus fuentes y sus audiencias (García-Avilés et. al 2009), porque "propicia la cooperación entre empresas de sectores distintos y la progresiva diversificación de soportes y canales de distribución por parte de los grandes grupos de comunicación". Se trata de un fenómeno que "afecta al trabajo de los profesionales y la organización de los medios y las redacciones, ya que ha desencadenado profundas transformaciones a la hora de concebir la información y el entretenimiento (GarcíaAvilés et. Al 2009).

Como explican Salaverría, García- Avilés y Masip (2008: 35), la convergencia periodística "no se agota en la mera integración redaccional". En la obra, los autores hablan de cierto miedo ante la concentración mediática, pero se trata de un miedo que no se percibe en las nuevas fusiones, cuyo objetivo no es exclusivamente económico, puesto que también se llevan a cabo para mejorar la calidad de los contenidos o simplemente a modo de colaboración. La diferencia fundamental entre el panorama de hace una década y el actual, la encontramos en la propia definición que ofrecen los autores de convergencia empresarial: "Cambios estructurales protagonizados por las grandes corporaciones de lo que podemos denominar industrias culturales, informáticas y de telecomunicaciones".

Como se verá en los ejemplos analizados, en la actualidad los cambios no solo los protagonizan las grandes corporaciones, sino también las nuevas iniciativas digitales que, aunque modestas, cuentan con un importante capital creativo. El informe titulado Punto de Inflexión. Impacto, amenazas y sustentabilidad: estudio de emprendedores de medios digitales latinoamericanos, llevado a cabo en 2017 por SembraMedia, en su apartado de recomendaciones, sugiere a las iniciativas construir puentes y alianzas para extender sus audiencias y compartir recursos. En este sentido, este informe también revela que las organizaciones de medios lideradas por mujeres son más cooperadoras, más proclives a formar alianzas y compartir recursos.

\section{Metodología}

Se estudia -mediante un análisis de casos y a través de entrevistas a los responsables de iniciativas innovadoras- la simbiosis entre diferentes medios para la creación de proyectos con más o menos continuidad; la absorción de unos por otros o las asociaciones y fusiones entre iniciativas o con organizaciones ajenas a la industria periodística. A continuación, se detalla una breve presentación de las dos páginas web 
sobre las que se asienta el análisis de casos de los medios llevado a cabo en el presente artículo:

Ranking de Innovación Periodística 2014 elaborado por la Universidad Miguel Hernández de Elche (UMH). Dicho ranking expone los proyectos periodísticos más innovadores en el ámbito español en 2014. Contempla las cuatro áreas principales del proceso periodístico: a) el producto o el servicio en sí mismo; b) los procesos de producción y distribución; c) la naturaleza de la organización o el equipo; d) y las acciones y estrategias de comercialización y marketing. El Ranking de innovación periodística fue publicado el 4 de diciembre de 2014 y fue elaborado por el siguiente equipo de investigadores profesores e investigadores de la $\mathrm{UMH}$ : Miguel Carvajal (diseño de la investigación); José Alberto García-Avilés (investigador senior); José Luis González Esteban (investigador senior); Alicia de Lara (coordinación y análisis); Félix Arias (algoritmos, datos y filtrado) y los investigadores juniores: José María Valero, Miguel Palazón y Borja García.

>http://mip.umh.es/ranking/index.html

El Observatorio de los Nuevos Medios, es un proyecto financiado por el programa de "Ayudas a Investigadores y creadores culturales 2015" de la Fundación BBVA, llevado a cabo por la profesora Marga Cabrera. Cabrera es doctora en Comunicación Audiovisual y profesora en la Universitat Politècnica de València, además de directora del Máster Universitario de Contenidos y Aspectos Legales en la Sociedad de la Información (CALSI). Coordinó el libro de la Fundéu BBVA "Escribir para internet: guía para los nuevos medios y las redes sociales", dirigido por Mario Tascón. Y es coautora, junto a Bárbara Yuste, del libro "Emprender en periodismo. Nuevas oportunidades para el profesional de la información", editado por la UOC.

$>$ http://www.nuevosmedios.es/

El universo de medios tenidos en cuenta parte de la consulta de ambos proyectos. En primer lugar, se han analizado los 25 medios que conforman el Ránking de Innovación Periodística 2014, poniendo el foco en los acuerdos y relaciones que existen entre ellos y con otros medios no contemplados en el ranking. En segundo lugar, para completar la muestra se consultó la página web del Observatorio de los Nuevos Medios. En un principio, se preguntó a los profesionales que se referencian a continuación a través de la entrevista sobre acuerdos y relaciones existentes entre medios e iniciativas periodísticas del panorama nacional, pero con el objetivo de contar con una muestra abundante que no se limitara a las relaciones existentes entre los medios mencionados por las fuentes consultadas, se consultó también las webs de los primeros 100 medios que ofrece la búsqueda en el proyecto limitada al panorama español. De nuevo se puso el foco en posibles relaciones con otras empresas y proyectos informativos dedicando entre 5 y 10 minutos (dependiendo de la complejidad de la web) a cada una de las páginas. 
Las entrevistas sobre las que se asientan los resultados del trabajo han sido realizadas por los profesores y alumnos colaboradores del Máster de Innovación de Periodismo de la UMH. Las cuestiones planteadas estaban relacionadas con conceptos relacionados con la innovación aplicada a las cuatro áreas principales del proceso periodístico: producto, distribución, comercialización y organización, siendo esta última área en la que se ha profundizado en el presente trabajo. El cuestionario contemplaba las siguientes 7 cuestiones:

1. ¿Qué entienden por innovar en periodismo?

2. ¿De qué modo tratan de innovar en la producción, organización, gestión, distribución y relación con la audiencia?

3. Formación para la innovación: con departamentos propios de innovación, fundaciones, universidades, entidades innovadoras (Medialab Prado...)

4. ¿Colaboradores externos? ¿Perfiles de los colaboradores externos? ¿Qué criterios priman en la contratación de nuevo personal?

5. Colaboraciones y acuerdos con otros proyectos: diferenciar entre las acciones conjuntas con otros medios

6. ¿Han recurrido a la investigación, consultoría o asesoramiento externo a la hora de innovar?

7. Número de personas que integran el proyecto sus perfiles

A continuación, se exponen los detalles de las 4 fuentes consultadas:

Alonso, Julio: fundador de Weblogs. Es autor del blog Merodeando, en el que trata temas relacionados con internet y el marketing digital. Durante seis años ha formado parte de la lista de El Mundo de las 25 personas más influyentes en internet en España. Entrevista realizada por Mari Carmen Borrull, octubre de 2016.

Belmonte, Eva: responsable de proyectos de la Fundación Civio. Alguno de los proyectos más conocidos de esta iniciativa son El BOE nuestro de cada día o Españopoly. También colabora con La Marea. Entrevista realizada por José María Valero en noviembre de 2015.

Espinosa, María Jesús: jefa de proyecto de Podium Podcast. Además, es la cofundadora de El Extrarradio, emisora de radio online ganadora del Premio Nacional Ondas 2013 a la Innovación Radiofónica. Colabora con otros medios como El País, Radio Nacional España o Jot Down. Entrevista realizada por Mari Carmen Borrull en enero de 2017.

Juan Luis Sánchez: cofundador y subdirector de eldiario.es. Está especializado en contenidos digitales y nuevos medios. También es uno de los fundadores de Periodismo Humano y fue su editor hasta 2012. Entrevista realizada por Lis Gaibar en noviembre de 2014 . 
El objetivo de esta investigación se centra en detectar y describir ejemplos representativos de las relaciones y acuerdos que se crean entre los medios de comunicación y las iniciativas periodísticas del panorama nacional. Se trata de un trabajo en progreso -no finalizado y que requiere una continua actualización- que pretende alcanzar un registro de alianzas en el panorama nacional no configurado hasta el momento y que ayuda a arrojar luz sobre el complejo y cambiante panorama mediático español.

\section{Resultados}

Son varios los casos en los que los nuevos medios interaccionan con otras iniciativas periodísticas nacionales e internacionales. Del estudio de las relaciones que se generan entre los medios digitales del panorama nacional, se pueden establecer tres tipos de relaciones que se detallan a continuación. De cada uno de los tipos señalados se incluyen ejemplos concretos detectados del análisis de los diferentes casos y sus principales características.

\subsection{Un nuevo proyecto que nace de la unión de otros proyectos}

En este primer tipo de relación entre medios se engloban aquellos casos en los que iniciativas que presentan cierta afinidad en su filosofía y contenidos se asocian para concebir un nuevo producto, fruto del trabajo de las distintas organizaciones colaboradoras. Tal y como se observa en los ejemplos descritos a continuación, no son muchos los casos en los que se percibe este tipo de vínculos. Encontramos tres casos, entre ellos un ejemplo del año 2013 que no perdura en la actualidad y otro caso actual que sí se presenta como un proyecto con futuro en el panorama mediático nacional.

En febrero de 2013, vio la luz el experimento editorial denominado Five, una selección de grandes y detallados artículos de cinco medios: Yorokobu, Diario Kafka, Naukas, Politikon y Jot Down Magazine. Cinco revistas que, tal y como se detallaba en aquel entonces eran "de reciente creación" y entendían el periodismo y la distribución de contenidos de una forma diferente a la visión tradicional de los medios convencionales. Se llegaron a publicar dos ediciones de esta revista.

El ejemplo más interesante de este tipo de relación mediática lo constituye Diagonal, ahora El Salto. Este proyecto colaborativo se basa en la creación de "un nuevo medio junto con 20 proyectos críticos. Un medio descentralizado y horizontal, un gran medio 
financiado por la gente", tal y como se explica en la propia web del proyecto. En la presentación de las líneas estratégicas de El Salto, que tuvo lugar en Madrid en noviembre de 2016, se explicó que el proyecto suponía "la creación de un nuevo medio de comunicación de calidad, incisivo, con nuevos formatos y hecho desde las premisas de la economía social".

El proyecto está formado, con distintos niveles de integración, por: Diagonal, Pikara Magazine, El Salmón Contracorriente, Arainfo (Aragón), Directa (Catalunya), La Marea, Praza Pública (Galiza), Último Cero (Valladolid), entre otros medios e iniciativas. Según sus impulsores, "El Salto es mucho más que una red de medios, puesto que constituye una apuesta por alcanzar una escala más grande gracias a una mirada construida colectivamente". Y su objetivo persigue la creación de una federación de medios en forma de cooperativa que reorganice el panorama mediático español y en el sur de Europa.

Otro proyecto en el que se encuentra implicado Diagonal, en este caso junto a La Marea es el monográfico impreso Cercanías. Consiste, según su web en "la primera publicación conjunta entre dos proyectos de referencia del periodismo autogestionado e independiente en el Estado español". Una sentencia con la que subraya la apuesta por el periodismo independiente alejándose de la relación con estamentos de poder. El artículo continúa explicando que estas dos iniciativas unen sus fuerzas con el siguiente objetivo de ofrecer información rigurosa y cercana al ciudadano. Es decir, una apuesta por periodismo local e independiente.

Como se puede apreciar, en los tres ejemplos se presentan proyectos que nacen del trabajo conjunto de diferentes medios, pero que no suponen la desaparición de los mismos.

\subsection{Colaboraciones}

El segundo tipo de relación entre iniciativas periodísticas es el que aglutina los diferentes ejemplos de colaboraciones con mayor o menor continuidad. Como se observa en el análisis de los casos, los medios pueden establecer acuerdos puntuales para llevar a cabo acciones concretas o colaborar entre sí, por ejemplo, para recibir formación o un servicio concreto en un campo específico en el que una de las dos iniciativas es puntera.

Un caso representativo de este tipo de colaboración es el evento Ciencia Jot Down, que, desde 2014, continúa celebrándose en Sevilla. En este certamen colabora estrechamente desde su puesta en marcha la revista de divulgación científica Naukas. La idea surgió precisamente en una de las primeras concentraciones de fans de la divulgación científica organizada por la publicación. 
Un ejemplo más fundamentado en la diversificación del negocio es el que presenta la Fundación Civio, que ha organizado seminarios para periodistas de medios tradicionales, como es el caso de El Mundo y El País. Pero también dirigidos a funcionarios públicos, tanto en Aragón como en la Administración General del Estado, con el objetivo de abordar la publicación de datos de una manera accesible, no solo para periodistas, sino también para ciudadanos. Eva Belmonte, responsable de proyectos de la Fundación Ciudadana Civio opina que lo más difícil a la hora de innovar en Periodismo es conseguir financiación. A su juicio, los modelos en los que está funcionando un sistema de socios potente tienen éxito porque "no solo venden esa información, sino que hacen sentir a los socios parte de una comunidad". En este sentido, la experta está aludiendo al marketing que llevan a cabo determinados medios digitales con el objetivo de crear comunidad, una estrategia documentada en estudios sobre medios como El Español (Doval-Avendaño, 2017) o el programa radiofónico Carne Cruda (Olmedo Salar y López Villafranca, 2018).

Por otra parte, Fíltrala es una plataforma independiente de denuncia ciudadana a través de la cual cualquier persona puede revelar información de interés público a medios de comunicación y organizaciones de la sociedad civil de manera segura y anónima. Entre los medios colaboradores con esta iniciativa destacan: Revista Mongolia, Critic, Directa, La Marea, Fundación Civio, Diagonal y eldiario.es. Otras organizaciones colaboradoras son: porCausa, La Plataforma en Defensa de la Libertad de Información (PDLI) y CEACCU, que es la primera organización de consumidores de España.

Las relaciones entre las iniciativas que constituyen este segundo tipo de colaboración mediática presentan un menor grado de fusión que las que se recogían en el tipo anterior. En esta ocasión, las iniciativas se relacionan entre sí no para crear contenido fruto del trabajo conjunto, sino para aprender, retroalimentarse u organizar actividades concretas afines a las audiencias de ambos medios.

En algunas ocasiones, como fue el caso de Jot Down Smart y El País la colaboración finalizó porque una de las partes quiso modificar los términos del contrato inicial para rediseñar un producto (Jot Down Smart) más afín al medio principal de distribución (EI País). Tras esta ruptura, Jot Down Smart funciona mediante suscripción.

\subsection{Asociaciones, fusiones y adquisiciones}

El tercer y último tipo de relación estudiada y que constituye la más común entre las iniciativas digitales de nuestra muestra de estudio es la que engloba tanto las asociaciones entre medios como las fusiones. 
El primer ejemplo de este tipo de relación se remonta a septiembre de 2014 y lo protagoniza el diario.es quien acoge bajo su paraguas al programa Carne Cruda: la república independiente de la radio, con el periodista Javier Gallego a la cabeza. Sin duda, una buena simbiosis, tanto por espíritu como por estar basados ambos en un modelo de negocio que apuesta por los socios y fans. Juan Luis Sánchez, cofundador y actual subdirector de eldiario.es defiende que "los modelos de innovación tienen sentido si uno conoce sus fortalezas y sus debilidades" y reivindica la importancia de un equipo multidisciplinar en el ámbito digital: "Hay que ser consciente de que el periodismo no sólo se hace con periodistas".

De nuevo eldiario.es destaca por incluir en algunas de sus secciones noticias publicadas tanto en blogs propios, como de otros proyectos asociados. Por ejemplo, contenidos de ámbito cultural, con Jot Down, o económico tecnológico, con HemerotekTecnoeconomía. Además, eldiario.es publica contenidos relacionados con los eventos promovidos por de la Revista Mongolia, incluyendo el enlace a la publicación.

En mayo de 2016, el portal web Bluper, especializado en noticias sobre televisión, se unió a El Español. Y en agosto de ese mismo año se produjo la fusión entre El Español y La Crónica, una alianza que el propio medio describió como "un ambicioso proyecto de suma de contenidos, cruce de participaciones y colaboración mutua a favor de los lectores". Asimismo, en noviembre de 2019, El Español se hace con el medio de información financiera de Teléfonica Invertia. En este caso no hablamos de una fusión, ni asociación, se trata de una adquisición de un millón de euros con la que el diario pretende incrementar su influencia en los ámbitos económicos del panorama nacional.

El caso de El Español requiere, de hecho, una mención aparte, puesto que se ha podido ver cómo en los últimos años ha apostado por adquirir varias cabeceras digitales, entre las que se encuentran, aparte de Blupper o La Crónica, otros títulos como Vandal y Omicrono. Una estrategia que le permitió en agosto de 2019 situarse como el sexto periódico digital más leído de España.

Por otra parte, la revista cultural conocida como El Asombrario se encuentra asociada al diario Público y, a su vez, concentra una serie de blogs como: Bonsauvage, Vías de Escape, Escuela de escritores y La sombra de Houdini, todos ellos en consonancia con los contenidos de la revista: arte y literatura, espectáculos, cine y música.

Asimismo, la fusión entre Contexto y Acción (CTXT) y Público se presenta como "medios amigos" a través de una alianza editorial. Las informaciones del semanario se difunden desde el 1 de junio el pasado año en este diario para enriquecer la oferta con contenidos "elaborados desde una perspectiva reposada y analítica". Según la web del propio diario Público, se lleva a cabo "una nueva colaboración editorial y publicitaria con la revista digital CTXT, con la que una y otra publicación pretenden revalorizar su 
oferta informativa, reforzar su presencia en las redes y afianzar su viabilidad económica". Asimismo, CTXT fue fundado en enero de 2015 por 14 periodistas con experiencia, procedentes de diarios como El País, El Mundo o La República, con la aspiración de ofrecer información más elaborada, a un ritmo pausado y con un enfoque analítico.

Otro ejemplo es la conocida empresa aglutinadora de blogs Weblogs, que se define como "el principal grupo de medios digitales especializados en español. Con presencia en España y en Latinoamérica". Gestiona más de 40 publicaciones propias que cada mes leen más de 55 millones de usuarios. Como explica el fundador de Weblogs, Julio Alonso, "los blogs tienen la ventaja de que abres uno y tienes la capacidad de llegar al público. De ahí la repercusión, aunque hay que trabajarlo mucho. La virtualidad acaba con el monopolio de los medios tradicionales ya que no pueden forzar a que la agenda informativa vaya por un solo lado. Ese papel de que los medios importantes vayan todos en una línea se ha roto, en parte, gracias a los blogs". El portal Weblogs se encuentra entre los 600 principales grupos de publicaciones en internet en el mundo, según los datos de ComScore. En España, se sitúa entre los 10 primeros grupos de publicaciones.

El último ejemplo de fusión de iniciativas lo constituye la plataforma de podcasting Podium Podcast, la red de contenidos sonoros de Prisa Radio, que fue galardonada con un Premio Ondas a la mejor plataforma radiofónica de emisión online en 2016. En esta ocasión, una misma web engloba en formato podcast los diferentes programas del conglomerado mediático, además de crear nuevos contenidos tanto en el terreno de la ficción como en el informativo. Al frente de la plataforma se encuentra María Jesús Espinosa, quien explica el éxito del proyecto como una fusión de la búsqueda de nuevos formatos y la recuperación de contenidos rescatados de la fonoteca del mayor grupo radiofónico en español.

Los diferentes casos analizados constituyen ejemplos de cómo los medios más innovadores establecen alianzas para alcanzar audiencias diversificadas, pero con intereses comunes ante ciertos contenidos.

Del estudio de las relaciones que se generan entre los medios digitales del panorama nacional, se pueden establecer tres tipos de relaciones atendiendo a tres parámetros que permiten sistematizar la clasificación: a) la duración o continuidad del acuerdo o colaboración; b) la afinidad de los contenidos y c) la afinidad existente entre las audiencias.

El primer tipo de relación hace referencia a aquellos casos en los que iniciativas que presentan cierta afinidad en su filosofía y contenidos se asocian para concebir un nuevo producto, fruto del trabajo de las distintas iniciativas colaboradoras. En este 
tipo de relaciones, entre las que se encontraba el caso de El Salto, se observan los siguientes parámetros:

a. Continuidad indefinida

b. Necesaria afinidad de contenidos

c. Necesaria afinidad de audiencias

El segundo tipo de relación entre iniciativas periodísticas es el que aglutina los diferentes ejemplos de colaboraciones. Como se observa en el análisis, los medios establecen acuerdos puntuales para llevar a cabo acciones concretas o que colaboran entre sí, por ejemplo, para recibir formación o un servicio concreto. Entre los ejemplos mencionados está la Fundación Civio ofreciendo servicios puntuales a otros medios. Vemos cómo, en esta ocasión, los parámetros se presentan con la siguiente combinación:
a. Continuidad esporádica
b. No es necesaria la afinidad de contenidos
c. No es necesaria la afinidad de audiencias

El tercer tipo de relación estudiada y que constituye la más común entre las iniciativas digitales analizadas es la que engloba tanto las asociaciones entre medios como las fusiones. En este caso también entrarían las adquisiciones, como el reciente caso de $E l$ Español comprando Invertia u otras adquisiciones más o menos recientes entre las que se podría mencionar el caso de Prisa adquiriendo Meristation, el Grupo Heraldo con La Información o eldiadio.es cuando se hizo con Vertele.

a. Continuidad estable

b. No es necesaria la afinidad de contenidos

c. No necesaria afinidad de audiencias, pero sí preferible dependiendo del caso

\section{Discusión y conclusiones}

Entre los ejemplos expuestos se descubren relaciones entre medios e iniciativas periodísticas de carácter muy diferente pero que resultan clasificables en tres categorías si se atiende a los tres parámetros descritos: continuidad o no de la relación; tipo de contenido y tipo de público al que se pretenden alcanzar. Las categorías definidas son: 1) Nuevo proyecto que nace de la unión de dos o más proyectos 2) Colaboraciones 3 ) Asociaciones, fusiones y adquisiciones.

Con esta propuesta pretendemos esbozar una clasificación que permita estudiar mejor el cambiante panorama de relaciones existente entre los medios e iniciativas periodísticas. Una propuesta de clasificación no cerrada y que necesita ser testada, pero que puede arrojar -ampliando el número de casos analizados- tendencias de 
interés sobre el comportamiento de los medios de comunicación en el terreno de las colaboraciones y su relación con la competencia.

En la mayoría de los ejemplos, además de los intereses de promoción y distribución, se pone de relieve la colaboración basada en la necesidad de alcanzar audiencias con intereses similares. También vemos cómo se asocian medios de diferente canal de distribución, como puede ser un diario nativo digital y un contenido radiofónico, algo que sucede en el caso de eldiario.es y el programa Carne Cruda. En este caso el nexo sobre el que se fundamenta la hermandad tiene un claro componente ideológico. $Y$, en este sentido, merece especial mención en el conjunto de casos expuestos el de Diagonal, que engloba a más de 20 medios de comunicación, de nuevo bajo un espíritu compartido de apuesta por lo social.

Estas son solo algunas de las alianzas que han tenido lugar en los últimos años entre iniciativas periodísticas que, por motivos estratégicos de diversa índole, han decidido fusionarse o colaborar, ya que el análisis se encuentra en proceso de ampliación, revisión y actualización constante. A pesar de tratarse de un work in progress, el trabajo consigue dejar patente que el conjunto de alianzas describe un interesante panorama que arroja un concepto de competencia diferente a la tradicional en la filosofía periodística de los nuevos medios.

A pesar de esta tendencia incipiente, el análisis también permite observar que las absorciones y adquisiciones son un tipo de relación entre medios con un gran peso. El caso de El Español es uno de los más representativos en este sentido, puesto que en los últimos años se ha hecho con diversas cabeceras digitales hasta alcanzar en verano de 2019 los 14,9 millones de visitantes únicos en Comscore, el medidor de referencia del mercado publicitario digital.

Es necesario señalar entre las limitaciones de la presente investigación que requiere de una actualización constante que garantice un seguimiento a los proyectos fruto de las relaciones entre medios, a las asociaciones entre iniciativas y a los acuerdos $y$ absorciones. Hoy por hoy, resulta imposible asegurar la viabilidad de algunos de los ejemplos mencionados porque las alianzas todavía son recientes. Llevar a cabo este seguimiento es una de las metas de este trabajo, porque consideramos que documentar de una manera sistematizada este conjunto de relaciones y analizar sus características podrá ofrecer a la investigación periodística pistas sobre la viabilidad de los proyectos y algunas claves para su supervivencia.

\section{Referencias bibliográficas}

Asociación de la Prensa de Madrid (APM) (2015): Informe Anual de la Profesión Periodística. Madrid. Recuperado de: https://www.apmadrid.es/noticiasdestacados/1-columna-arriba-2/informe-anual-de-la-profesion-periodistica2015 ? Itemid $=$

Asociación de la Prensa de Madrid (APM) (2018): Informe Anual de la Profesión Periodística. Madrid. Recuperado de: 
https://cdn27.hiberus.com/uploads/documentos/2018/12/10/_informeanual20 18baja1_3d6ba497.pdf

BORCHARDT, A., LÜCK, J., KIESLICH, S., SCHULTZ, T., \& SIMON, F. M. (2019): Are Journalists Today's Coal Miners? Oxford University and Reuters Institute. Oxford and Mainz https://reutersinstitute.politics.ox.ac.uk/sites/default/files/201907/Talent-and-Diversity-in-the-Media-Report_0.pdf

BOCZKOWSKI, P. J. (2006): Digitalizar las noticias: Innovación en los diarios online. Buenos Aires. Ediciones Manantial.

BRIGGS., M. (2012): Entrepreneurial Journalism. London: Sage

BECERRA, M. (2000): "De la divergencia a la convergencia en la sociedad informacional: fortaleza y debilidades de un proceso inconcluso". Revista de Estudios de Comunicación ZER, núm.8, pp. 93-112. Disponible en: http://www.ehu.eus/ojs/index.php/Zer/article/view/17418

CABRERA GONZÁLEZ, M. Á. (2010): Evolución tecnológica y cibermedios (Vol. 34). Sevilla-Zamora. Comunicación Social.

CANAVILHAS, J. (2009): “Contenidos informativos para móviles: estudio de aplicaciones para iPhone". Textual \& visual media, (2), 61-80. Disponible en: http://www.bocc.ubi.pt/pag/canavilhas-joao-contenidos-informativos-paramoviles.pdf

CEBRIÁN HERREROS, M. (2010): Desarrollos del periodismo en Internet (Vol. 37). Sevilla-Zamora. Comunicación Social.

De LARA GONZÁLEZ, CARVAJAL PRIETO, M., GARCÍA-AVILÉS, J. A., \& Arias Robles, F. (2014): Innovar más allá del producto periodístico. El caso de las 25 iniciativas del Ranking de Innovación Periodística en España 2014. Conference: XIII Congreso de Periodismo Digital de Huesca.

DE LARA GONZÁLEZ, A., ÁRIAS-ROBLES, F., CARVAJAL-PRIETO, M., \& GARCÍA-AVILÉS, J. A. (2015): "Ranking de Innovación Periodística en España 2014. Selección y análisis de 25 iniciativas". El profesional de la información, 24(3). Disponible en: https://recyt.fecyt.es/index.php/EPI/article/download/epi.2015.may.02/19952

DOMINGO, D. (2005): Rutinas profesionales y valores en las redacciones de medios digitales catalanes: periodismo digital en contextos reales. En II Congreso Online del Observatorio de la Cibersociedad.

DOGRUEL, L. (2013): "Opening the Black Box - Conceptualizing of Media Innovation". En S. Storsul \& A. H. Krumsvik (eds.), Media Innovation. A Multidisciplinary Study of Change. Göteborg: Nordicom, 29-44. 
DOVAL-AVENDAÑO, M. (2017): La creación de comunidad en Twitter alrededor de un medio inexistente, El Español. Estudios sobre el Mensaje Periodístico, 23(1), 391.

FLORES VIVAR, J. M. (2009): Nuevos modelos de comunicación, perfiles y tendencias en las redes sociales. Comunicar, no 33, v. XVII, 2009, Revista Científica de Educomunicación; ISSN: 1134-3478; páginas 73-81 Disponible en http://rabida.uhu.es/dspace/handle/10272/2843

GARCÍA-AVILÉS, J. A., CARVAJAL-PRIETO, M., De LARA-GONZÁLEZ, A., \& ARIAS-ROBLES, F. (2016): Developing an Index of Media Innovation in a National Market: The case of Spain. Journalism Studies, 1-18.

GARCÍA-AVILÉS, J. A.; SALAVERRÍA, R., y MASIP, P. (2008): Convergencia periodística en los medios de comunicación. Propuesta de definición conceptual y operativa, en: I Congreso de la Asociación Española de Investigadores en Comunicación, Santiago de Compostela.

GARCÍA-AVILÉS, J. A. G. (2009): La comunicación ante la convergencia digital: algunas fortalezas y debilidades. Signo y pensamiento, 28(54), 102-113. Disponible en: https://revistas.javeriana.edu.co/index.php/signoypensamiento/article/view/45 29

García-AVILÉS, J. A. G., PRieto, M. C., KAltenkRUnNeR, A., MEIER, K., \& KRAUS, D. (2009): Integración de redacciones en Austria, España y Alemania: modelos de convergencia de medios. Anàlisi: quaderns de comunicació i cultura, (38), 173198. Disponible en: http://dspace.umh.es/bitstream/11000/4559/1/14convergencia\%20de\%20medios.pdf

GARCÍA-AVILÉS, J. A. G. (2016): La información ya no es lo que era". En Diez propuestas sobre el periodismo en la Sociedad Red. RAE-IC, $\mathrm{p}$ 1-8. Disponible en: http://www.revistaeic.eu/index.php/raeic/article/view/114

GARCíA-SANTAMARÍA, J. V., Fernández, C., Dolores, M., \& López Aboal, M. U. (2013). "La organización de las redacciones en los nuevos diarios digitales españoles y su relación con los nuevos modelos de negocio". Textual \& Visual Media 6, 2013. Disponible en: http://www.textualvisualmedia.com/index.php/txtvmedia/article/view/81

JENKINS, H.(2008). Convergence culture. La cultura de la convergencia de los medios de comunicación, Barcelona, Paidós.

MESO AYERDI, K. (2013). "Periodismo y audiencias: inquietudes sobre los contenidos generados por los usuarios". Cuadernos. info, (33), 63-73. Disponible en: https://scielo.conicyt.cl/scielo.php?pid=S0719367X2013000200006\&script=sci_arttext 
OLMEDO SALAR, S. y López Villafranca, P. (2018). Nuevos medios independientes en el panorama mediático digital. Las emisoras de radio online en España: el caso de Carne Cruda y Radiocable. index.comunicación, 8(3), 135-155. Disponible en: http://journals.sfu.ca/indexcomunicacion/index.php/indexcomunicacion/article/ view/422/430

PASTOR, J. M. V. (2015): Tendencias de la innovación mediática en Estados Unidos. Miguel Hernández Communication Journal, (6), 161-193. Disponible en: https://dialnet.unirioja.es/servlet/articulo?codigo $=5281775$

PUERTA, S. V. (2006): Las estructuras, la cultura y el clima organizacional en la empresa informativa: la tensión entre la administración y el valor periodístico. NOVUM: Revista de Ciencias Sociales Aplicadas, (31), 77. Disponible en: https://revistas.unal.edu.co/index.php/novum/article/view/45655

SALAVERRÍA, R. (2009): Los medios de comunicación ante la convergencia digital, Actas del I Congreso Internacional de Ciberperiodismo y Web 2.0 (CD-Rom), Bilbao, 11-13 noviembre 2009. Servicio Editorial de la Universidad del País Vasco. ISBN 978-84-9860-058-2.

SembraMedia (2017): Punto de inflexión. Impacto, amenazas y sustentabilidad: estudio de emprendedores de medios digitales latinoamericanos. Recuperado de: http://data.sembramedia.org/wp-content/uploads/2017/09/Punto-de-InflexionSembraMedia-span-7-24.pdf

STEEN STEENSEN (2009): What's stopping them? Towards a grounded theory of innovation in online journalism. Journalism Studies, 10, 6, 821-836

TÚÑEZ, M. (2009): Jóvenes y prensa en papel en la era Internet. Estudio de hábitos de lectura, criterios de jerarquía de noticias, satisfacción con los contenidos informativos y ausencias temáticas. Estudios sobre el mensaje periodístico, 15, 503-525. Disponible en: https://core.ac.uk/download/pdf/38814119.pdf 